\title{
USO DE COMPOSTA, MINERALES PRIMARIOS AMORFOS Y MICROORGANISMOS PARA LA PRODUCCIÓN Y CALIDAD DE TOMATE
}

\author{
USE OF COMPOST, PRIMARY AMORPHOUS MINERALS AND MICROORGANISMS FOR \\ PRODUCTION AND QUALITY OF TOMATO
}

\author{
Juan Martín Parra-Delgado, Teresa de J. Velázquez-Alcaraz, Edgar Quero-Gutiérrez, \\ Leopoldo Partida-Ruvalcaba, Tomás Díaz-Valdés, Blas Galván-Piña y Felipe Ayala-Tafoya
}

\begin{abstract}
RESUMEN
La presente investigación se realizó con el objetivo de determinar el efecto de la composta bocashi, minerales primarios amorfos y microorganismos en el rendimiento y calidad postcosecha de tomate tipo saladette (Lycopersicum esculentum Mill.), híbrido "Moctezuma". El cultivo se realizó en suelo bajo condiciones de invernadero, durante el ciclo agrícola otoño-invierno 2011-2012. Se utilizó una densidad de población de 2,5 plantas $\mathrm{m}^{2}$, manejadas a un tallo y con una separación de 1,6 m entre hileras. La composta bocashi, los minerales primarios amorfos y los microorganismos fueron incorporados al suelo 30 días antes del inicio del cultivo, y estos últimos también se aplicaron después del trasplante. Se evaluó el rendimiento total y de tomates extra grandes, grandes, medianos y chicos de ocho racimos por planta, así como su calidad postcosecha. Para las variables de rendimiento se consideró un diseño experimental de bloques completos al azar conformado por nueve tratamientos, mientras que para el estudio de calidad postcosecha se utilizó un diseño completamente al azar con los mismos tratamientos. El mayor rendimiento $\left(113,97 \mathrm{Mg} \mathrm{ha}^{-1}\right)$ se obtuvo con el tratamiento compuesto por 25 y $6 \mathrm{Mg}^{-1} \mathrm{de}^{-1}$ composta y minerales primarios amorfos, respectivamente, el cual superó en 9,3 \% a los rendimientos logrados solo con la solución Steiner, aunque sin diferencia estadísticamente significativa entre ambos promedios. Los tratamientos no tuvieron ningún efecto en el pH, la acidez titulable y los sólidos solubles totales.
\end{abstract}

PALABRAS CLAVE: suelos arcillosos, compostaje, consorcio microbiano, solución Steiner, cosechas

\begin{abstract}
This research was carried out with the aim to determine the influence of the bocashi compost, amorphous primary minerals and microorganisms in post-harvest yield and quality of saladette tomato (Lycopersicum esculentum Mill.) hibrid "Moctezuma". The crop was carried in soil under greenhouse conditions during the agricultural cycle 2011-2012 autumn-winter. A population density of 2.5 plants $\mathrm{m}^{2}$ managed to a single stem and a separation of $1.6 \mathrm{~m}$ between rows was used. The bocashi compost and amorphous primary minerals were incorporated into the soil 30 days before the start of cultivation. Total and extra-large, large, medium and small tomatoes yields of eight clusters per plant and their postharvest quality were evaluated. An experimental design of randomized complete blocks with nine treatments was applied for yield variables, while a completely randomized design was used for postharvest quality variables. The highest yield (113.97 $\mathrm{Mg} \mathrm{ha}^{-1}$ ) was obtained with the treatment consisting of 25 and $6 \mathrm{Mg} \mathrm{ha}^{-1}$ of compost and amorphous primary minerals, respectively, which exceeded $9.3 \%$ those achieved with the Steiner solution based treatments, although no significant statistically difference were found between the two averages. $\mathrm{pH}$, titratable acidity and total soluble solids of fruits were not significantly affected by treatments.
\end{abstract}

KEY WORDS: clay soils, composting, microbial consortium, Steiner solution, crops

102

Dirección de los autores:

Facultad de Agronomía de la Universidad Autónoma de Sinaloa Carretera Culiacán-Eldorado Km. 17.5. Apdo. Postal 726. Culiacán, Sinaloa, México. (J.M.P.D.) (T.J.V.A.) (T.D.V.) (B.G.P.) (F.A.T.). Colegio de Michoacán. Laboratorio de Análisis y Diagnóstico del Patrimonio. Cerro de Nahuatzen 85. Frac. Jardines del Cerro Grande. C.P. 59370. La Piedad, Michoacán, México (E.Q.G.). Universidad Tecnológica de Culiacán, Carretera CuliacánImala km 2, Col. Los Ángeles, C. P. 80014, en la Ciudad Educadora del Saber, Culiacán Rosales, Sinaloa, México (L.P.R.). E-mail martinparra2004@ yahoo.es (J.M.P.D.) 


\section{INTRODUCCIÓN}

El uso excesivo de fertilizantes inorgánicos de N, P y K en la agricultura moderna ha provocado la degradación de los suelos y la contaminación de mantos freáticos y la atmósfera (Villarreal et al., 2006). En México, el contenido de materia orgánica en los suelos es bajo (Ortiz y Amado, 2003). Lo anterior ha provocado que la agricultura sea cada vez más dependiente de agroinsumos. El uso de composta es una alternativa para la producción de cultivos, ya que mejora una gran cantidad de características del suelo como la fertilidad, la densidad aparente, la capacidad de almacenamiento de agua, la capacidad de intercambio catiónico y la mineralización del nitrógeno, el fósforo y potasio. Además, disminuye el lavado de nitratos, mantiene valores de $\mathrm{pH}$ óptimos para la agricultura, evita cambios extremos en la temperatura, fomenta la actividad microbiana, reduce microorganismos patógenos, controla la erosión y fomenta la eliminación de semillas de malezas por las altas temperaturas generadas por la actividad microbiana durante el composteo y la degradación de plaguicidas (Nieto et al., 2002). Se deben aprovechar los recursos existentes en cada región para insertarlos en los ciclos de producción, evitar que se conviertan en fuentes de contaminación de mantos freáticos y del suelo (Olivares et al., 2012). Los abonos orgánicos pueden satisfacer la demanda de nutrientes de los cultivos, reduciendo significativamente el uso de fertilizantes químicos (Rodríguez et al., 2009) y mejorando la fertilidad del suelo, por el efecto que genera la biodiversidad microbiana, que actúan conjuntamente como una comunidad en un sistema complejo donde todos se benefician (Ochoa y Montoya, 2010). A lo anterior también se suma el creciente interés por utilizar fuentes alternas de nutrimentos, como las rocas silicatadas que varían en su disponibilidad y potencial de liberación de nutrientes (Da Silva et al., 2010), además de aumentar la fertilidad del suelo (Guelfi et al., 2012). La utilización de roca pulverizada es un proceso práctico que reduce el consumo de energía y aumenta la disponibilidad de nutrientes al combinarse con microorganismos que solubilizan los minerales primarios amorfos (MPA) (Stamford et al., 2009).

El tomate es la segunda hortaliza que más se produce en el mundo (aproximadamente 164 millones de t), correspondiendo a México el $2 \%$ de la producción mundial. En Sinaloa (México) las dosis de fertilizantes que se aplican están por encima de los requerimientos del cultivo, como es el caso del nitrógeno, que fluctúa entre 350 y $400 \mathrm{~kg} \mathrm{ha}^{-1}$ (Villarreal et al., 2006). La nutrición de esta hortaliza se puede satisfacerse con composta, MPA y microorganismos con un mínimo impacto ambiental y mayor propiedad energética (Nicholls y Altieri, 2012).

El objetivo de la presente investigación fue determinar el efecto que ocasionan la composta bocashi, los MPA y los microorganismos en la producción y calidad postcosecha de tomate cultivado en suelo.

\section{MATERIALES Y MÉTODOS}

El trabajo experimental se realizó en un invernadero de la Facultad de Agronomía de la Universidad Autónoma de Sinaloa, ubicado en el kilómetro 17,5 de la carretera Culiacán-Eldorado (24 $48^{\prime} 28^{\prime \prime}$ latitud norte y $107^{\circ}$ 24' 30" longitud oeste), en un suelo vertisol crómico. Según García (1988) el clima es semiseco, muy cálido, extremoso, con lluvias en verano, con temperatura media anual de $25,9^{\circ} \mathrm{C}$ y precipitación media anual de $772 \mathrm{~mm}$.

Se utilizó el híbrido 'Moctezuma' de tomate saladette (Lycopersicum esculentum Mill., 1768) con hábito de crecimiento indeterminado, el cual se cultivó en surcos separados a 1,6 $\mathrm{m}$ y una densidad de población de 2,5 plantas $\mathrm{m}^{2}$. El aporte de agua y nutrientes se realizó a través de un sistema de riego por goteo y en cada surco se colocó doble manguera con goteros cada $30 \mathrm{~cm}$. El trasplante se realizó el 12 de diciembre de 2011 y las plantas se manejaron a un tallo.

Se estableció el experimento bajo un diseño de bloques completos al azar con cuatro repeticiones y los tratamientos indicados en la Tabla 1, los cuales se definieron con base en lo recomendado por Steiner (1961) y teniendo en cuenta que la composta contiene de 1-3 \% de N, con mineralización del 18 \% (Egbahall, 2002), mientras que el consorcio microbiano y los MPA se formularon en base a lo recomendado por los distribuidores.

La composta bocashi (rastrojo de maíz, estiércol de bovino, salvado de arroz, cascarilla de arroz, melaza, levadura de pan, carbón vegetal triturado, tierra de aluvión y agua) y los MPA se aplicaron al suelo un mes (ADT), manteniéndose a capacidad de campo durante 30 días; posteriormente el suelo se removió y mezcló antes del trasplante (ADT). La solución Steiner se aplicó desde el trasplante hasta el fin de cosecha, en 
tanto que en las parcelas donde se asignaron los otros tratamientos sólo se aplicó agua, y los microorganismos se aplicaron en los tratamientos correspondientes cada ocho días a través del riego. Las variables de respuesta fueron la producción total (considerándose la cosecha de ocho racimos por planta), y la producción de frutos clasificada en extra grande ( $>150 \mathrm{~g}$ ), grande (125-149 g), mediano (100-124 g) y chico (75-99 g) (USDA, 1992).

Para la variable de calidad postcosecha se utilizó un diseño completamente al azar con cuatro repeticiones y los mismos tratamientos que se describen en la Tabla 1. Se determinó $\mathrm{pH}$, acidez titulable y sólidos solubles totales ( ${ }^{\circ}$ Brix). Los frutos fueron cosechados a los 93 DDT, en grado dos de madurez fisiológica, se lavaron con agua clorada a $100 \mathrm{mg} \mathrm{kg}^{-1}$ y se secaron a temperatura ambiente; posteriormente se almacenaron a $20^{\circ} \mathrm{C}$ y 85 $\%$ de humedad relativa, y las evaluaciones se realizaron a los cero, ocho y dieciséis días de almacenamiento. Para el pH y la acidez titulable se utilizó un titulador automático DL 50 (Metler, Toledo), se disolvieron 10 $\mathrm{g}$ de muestra en $50 \mathrm{ml}$ de agua destilada a $\mathrm{pH}=7,0$. Posteriormente se filtró y se obtuvo el extracto, del cual se tomaron $20 \mathrm{ml}$ y se tituló con $\mathrm{NaOH} 0,1 \mathrm{~N}$ hasta pH de 8,2. Para los sólidos solubles totales se utilizó un refractómetro RE 40D (Metler Toledo) donde se colocaron gotas del extracto y se tomó la lectura (AOAC, 1975). Los valores de $\mathrm{pH}$, acidez titulable y sólidos solubles totales se reportaron como iones de hidrógeno, porcentaje de ácido cítrico y ${ }^{\circ}$ Brix, respectivamente.

Tabla 1. Tratamientos utilizados para la producción de tomate saladette en invernadero. Ciclo agrícola 2011-2012. Culiacán, Sinaloa (México)

\begin{tabular}{|c|c|c|}
\hline Tratamientos y control & Abreviatura & Sustancias/mezclas \\
\hline Control (Tratamiento 1) & $\mathrm{T}_{1}$ & Solución Steiner 100 \% \\
\hline Tratamiento 2 & $\mathrm{~T}_{2}$ & $15 \mathrm{Mg} \mathrm{ha}^{-1}$ composta bocashi $+3 \mathrm{Mg} \mathrm{ha}^{-1} \mathrm{MPA}$ \\
\hline Tratamiento 3 & $\mathrm{~T}_{3}$ & $15 \mathrm{Mg} \mathrm{ha}^{-1}$ composta $+3 \mathrm{Mg} \mathrm{ha}^{-1} \mathrm{MPA}+2 \mathrm{~L} \mathrm{ha}^{-1}$ de $\mathrm{M}$ \\
\hline Tratamiento 4 & $\mathrm{~T}_{4}$ & $15 \mathrm{Mg} \mathrm{ha}^{-1}$ composta $+6 \mathrm{Mg} \mathrm{ha}^{-1} \mathrm{MPA}$ \\
\hline Tratamiento 5 & $\mathrm{~T}_{5}$ & $15 \mathrm{Mg} \mathrm{ha}^{-1}$ composta $+6 \mathrm{Mg} \mathrm{ha}^{-1} \mathrm{MPA}+2 \mathrm{~L} \mathrm{ha}^{-1}$ de M \\
\hline Tratamiento 6 & $\mathrm{~T}_{6}$ & $25 \mathrm{Mg} \mathrm{ha}^{-1}$ composta $+3 \mathrm{Mg} \mathrm{ha}^{-1} \mathrm{MPA}$ \\
\hline Tratamiento 7 & $\mathrm{~T}_{7}$ & $25 \mathrm{Mg} \mathrm{ha}^{-1}$ composta $+3 \mathrm{Mg} \mathrm{ha}^{-1} \mathrm{MPA}+2 \mathrm{~L} \mathrm{ha}^{-1}$ de M \\
\hline Tratamiento 8 & $\mathrm{~T}_{8}$ & $25 \mathrm{Mg} \mathrm{ha}^{-1}$ composta $+6 \mathrm{Mg} \mathrm{ha}^{-1} \mathrm{MPA}$ \\
\hline Tratamiento 9 & $\mathrm{~T}_{9}$ & $25 \mathrm{Mg} \mathrm{ha}^{-1}$ composta $+6 \mathrm{Mg} \mathrm{ha}^{-1} \mathrm{MPA}+2 \mathrm{~L} \mathrm{ha}^{-1}$ de $\mathrm{M}$ \\
\hline
\end{tabular}

MPA: Minerales Primarios Amorfos

M: Microorganismos

Los resultados de producción fueron sometidos al análisis de varianza y comparaciones múltiples de medias con la prueba de Duncan $(\alpha=0,05)$, mientras que a los valores de calidad postcosecha se les realizó análisis de varianza y para la separación de medias se empleó la prueba de Tukey $(\mathrm{p} \leq 0,05)$, mediante el programa SAS Versión 5 (1985).

\section{RESULTADOS}

104

Con el tratamiento $8\left(\mathrm{~T}_{8}\right)$ se obtuvo una producción de tomate extra grande sin diferencia estadística con respecto a la que se obtuvo con la solución Steiner, pero superó en $155,3,198,6,152,8,300,2,136,4,250,5$ y $46,1 \%$ a los tratamientos $\mathrm{T}_{2}, \mathrm{~T}_{3}, \mathrm{~T}_{4}, \mathrm{~T}_{5}, \mathrm{~T}_{6}, \mathrm{~T}_{7} \mathrm{y} \mathrm{T}_{9}$, respectivamente. Sin embargo, con la solución Steiner $\left(\mathrm{T}_{1}\right)$ los incrementos fueron de 174,2, 220,7, 171,5, 329,8, 153,9, 276,4, 7,4 y $56,9 \%$ (Tabla 3 ). Con respecto al tamaño grande, $\mathrm{T}_{8}$ ocasionó una producción que estadísticamente sólo superó a lo cosechado en las parcelas fertilizadas con $\mathrm{T}_{3}, \mathrm{~T}_{5} \mathrm{y} \mathrm{T}_{7}$ (diferencias de 44,2, 39,9 y 46,2\%, respectivamente). Con relación a los frutos de tamaño mediano, se cosecharon $27,1 \mathrm{Mg}^{-1}{ }^{-1}$ con el tratamiento $\mathrm{T}_{2}$, superando estadísticamente la producción obtenida mediante los tratamientos $\mathrm{T}_{1}, \mathrm{~T}_{5}$ y $_{9}$ (diferencias de 62,9, 56,8 y $40,2 \%$, respectivamente) (Tabla 3 ). 
Tabla 2. Características físico-químicas de la composta y minerales primarios amorfos y concentraciones utilizadas de las especies del consorcio microbiano

\begin{tabular}{|c|c|c|c|c|c|}
\hline Característica & Composta & Característica & $\begin{array}{c}\text { Minerales } \\
\text { primarios } \\
\text { amorfos }\end{array}$ & Consorcio microbiano & Concentración \\
\hline $\mathrm{pH}$ & 9.30 & $\mathrm{pH}$ & 8.9 & Bacillus subtilis & $1 \times 10^{18} \mathrm{ufc} \mathrm{ml}^{-1}$ \\
\hline $\mathrm{CE}\left(\mathrm{dS} \mathrm{m}^{-1}\right)$ & 12.0 & $\mathrm{CE}\left(\mathrm{dS} \mathrm{m}^{-1}\right)$ & 4.5 & Trichoderma harzianum & $2 \times 10^{8} \mathrm{ufc} \mathrm{g}^{-1}$ \\
\hline MO (\%) & 10.78 & $\mathrm{SiO}_{2}(\%)$ & 34 & Azotobacter spp & $1 \times 10^{6}$ ufc g $^{-1}$ \\
\hline N-total (\%) & 0.78 & $\mathrm{P}_{2} \mathrm{O}_{5}(\%)$ & 9.6 & Azozpirillum brasilense & $1 \times 10^{6} \mathrm{ufc} \mathrm{g}^{-1}$ \\
\hline $\mathrm{P}_{2} \mathrm{O}_{5}(\%)$ & 0.34 & $\mathrm{~K}_{2} \mathrm{O}(\%)$ & 2.5 & Glomus intraradices & 20 esporas por $\mathrm{g}^{-1}$ \\
\hline $\mathrm{K}_{2} \mathrm{O}(\%)$ & 1.90 & $\mathrm{CaO}(\%)$ & 13 & & \\
\hline Ca $(\%)$ & 1.48 & $\operatorname{MgO}(\%)$ & 2.0 & & \\
\hline $\operatorname{Mg}(\%)$ & 0.93 & $\mathrm{Fe}\left(\mathrm{mg} \mathrm{kg}^{-1}\right)$ & 18 & & \\
\hline $\mathrm{Fe}\left(\mathrm{mg} \mathrm{kg}^{-1}\right)$ & 1.9 & $\mathrm{Zn}\left(\mathrm{mg} \mathrm{kg}^{-1}\right)$ & 16 & & \\
\hline $\mathrm{Cu}\left(\mathrm{mg} \mathrm{kg}^{-1}\right)$ & 16 & $\operatorname{Mn}\left(\mathrm{mg} \mathrm{kg}^{-1}\right)$ & 5 & & \\
\hline $\mathrm{Zn}\left(\mathrm{mg} \mathrm{kg}^{-1}\right)$ & 65 & & & & \\
\hline $\mathrm{Mn}\left(\mathrm{mg} \mathrm{kg}^{-1}\right)$ & 390 & & & & \\
\hline $\mathrm{B}\left(\mathrm{mg} \mathrm{kg}^{-1}\right)$ & 120 & & & & \\
\hline
\end{tabular}

Tabla 3. Producción de tomate saladette (extra grande, grande, mediano, chico y producción total), con el uso de solución Steiner, composta, minerales primarios amorfos y microorganismos

\begin{tabular}{cccccc}
\hline Tratamientos & $\begin{array}{c}\text { Extra grande } \\
\left(\mathbf{M g ~ h a} \mathbf{~}^{-1}\right)\end{array}$ & $\begin{array}{c}\text { Grande } \\
\left(\mathbf{M g ~ h a}^{-1}\right)\end{array}$ & $\begin{array}{c}\text { Mediano } \\
\left(\mathbf{M g ~ h a}^{-1}\right)\end{array}$ & $\begin{array}{c}\text { Chico } \\
\left(\mathbf{M g ~ h a}^{-1}\right)\end{array}$ & $\begin{array}{c}\text { Total } \\
\left(\mathbf{M g ~ h a}^{-1}\right)\end{array}$ \\
\hline $\mathrm{T}_{1}$ & $50,117 \mathrm{a}^{*}$ & $32,184 \mathrm{ab}$ & $16,630 \mathrm{~b}$ & $5,351 \mathrm{~d}$ & $\begin{array}{c}104,282 \mathrm{ab} \\
92,641 \mathrm{bc}\end{array}$ \\
$\mathrm{T}_{2}$ & $18,280 \mathrm{c}$ & $32,496 \mathrm{ab}$ & $27,084 \mathrm{a}$ & $14,781 \mathrm{a}$ & $72,505 \mathrm{~cd}$ \\
$\mathrm{~T}_{3}$ & $15,626 \mathrm{c}$ & $23,068 \mathrm{c}$ & $20,534 \mathrm{ab}$ & $13,278 \mathrm{abc}$ & $79,972 \mathrm{~cd}$ \\
$\mathrm{~T}_{4}$ & $18,460 \mathrm{c}$ & $26,293 \mathrm{abc}$ & $21,617 \mathrm{ab}$ & $13,603 \mathrm{ab}$ & $65,505 \mathrm{~d}$ \\
$\mathrm{~T}_{5}$ & $11,660 \mathrm{c}$ & $23,775 \mathrm{bc}$ & $17,277 \mathrm{~b}$ & $12,793 \mathrm{abc}$ & $80,923 \mathrm{~cd}$ \\
$\mathrm{~T}_{6}$ & $19,739 \mathrm{c}$ & $27,113 \mathrm{abc}$ & $21,239 \mathrm{ab}$ & $12,834 \mathrm{abc}$ & $73,839 \mathrm{~cd}$ \\
$\mathrm{~T}_{7}$ & $13,313 \mathrm{c}$ & $22,743 \mathrm{c}$ & $23,113 \mathrm{ab}$ & $14,671 \mathrm{a}$ & $113,970 \mathrm{a}$ \\
$\mathrm{T}_{8}$ & $46,668 \mathrm{a}$ & $33,255 \mathrm{a}$ & $23,141 \mathrm{ab}$ & $10,905 \mathrm{bc}$ & $10,584 \mathrm{c}$ \\
$\mathrm{T}_{9}$ & $31,942 \mathrm{~b}$ & $28,187 \mathrm{abc}$ & $19,313 \mathrm{~b}$ & $90,025 \mathrm{bc}$ \\
\hline
\end{tabular}

${ }^{*}$ Medias con la misma letra en la columna no son significativamente diferentes, Duncan $(\mathrm{P}>0,05)$.

En la misma tabla se denota que con $\mathrm{T}_{2}$ y $\mathrm{T}_{7}$ se cosecharon, respectivamente, 14,8 y $14,7 \mathrm{Mg}^{-1}$ de tomate de tamaño chico, de tal forma que con $\mathrm{T}_{2}$ el rendimiento superó en 176,2, 35,5 y 39,6 \% los obtenidos con $\mathrm{T}_{1}, \mathrm{~T}_{8}$ y $\mathrm{T}_{9}$, respectivamente. Con $\mathrm{T}_{7}$ los incrementos fueron de 174,2, 34,5 y 38,6 \%. La mayor producción total (113,97 Mg ha-1) se logró con el $\mathrm{T}_{8}$, que incluyó $26 \mathrm{Mg}$ ha-1 de composta y $6 \mathrm{Mg}^{-1} \mathrm{ha}^{-1}$ de MPA. Dicha producción superó en $23,0,57,2,42,5,74,50$, $40,8,54,3$ y $26,6 \%$ las obtenidas, en su orden, con $\mathrm{T}_{2}, \mathrm{~T}_{3}, \mathrm{~T}_{4}, \mathrm{~T}_{5}, \mathrm{~T}_{6}, \mathrm{~T}_{7} \mathrm{y} \mathrm{T}_{9}$. Sin embargo, el promedio de $113,97 \mathrm{Mg} \mathrm{ha}^{-1}$ fue estadísticamente igual al de 104,282 $\mathrm{Mg} \mathrm{ha}^{-1}$ que se obtuvo en las parcelas fertilizadas con la solución Steiner. 
Con relación a los análisis químicos de postcosecha (día cero) el pH de los frutos disminuyó en un 4,4 \% en los tomates cosechados mediante el tratamiento $\mathrm{T}_{4} \mathrm{y} 4,7 \%$ en aquéllos cultivados en el $\mathrm{T}_{5}$, mientras que con $\mathrm{T}_{8}$ la disminución fue del 6,2 \% en relación a los cosechados de las plantas que recibieron la solución Steiner (testigo) (Tabla 4). A los ocho días de almacenados también se observó variación del pH, pero éste tuvo incrementos de 2,5 \% sólo en aquellos frutos que fueron cosechados de plantas tratadas con $\mathrm{T}_{5} \mathrm{y} \mathrm{T}_{8}$, en comparación con el promedio del testigo; sin embargo, a los 16 días el $\mathrm{pH}$ de los frutos ya fue igual para todos los tratamientos. En la misma tabla se puede observar que la acidez titulable no varió a los cero u ocho días de almacenamiento, pero a los 16 días dicha acidez se incrementó $28 \%$ en los frutos de plantas cultivadas con $\mathrm{T}_{3} \mathrm{y} \mathrm{T}_{4}$, en comparación con la acidez en frutos de plantas manejadas con $\mathrm{T}_{7} \mathrm{y}$ $\mathrm{T}_{8}$, sin que dicha característica en las plantas cultivadas con $\mathrm{T}_{3}$ y $\mathrm{T}_{4}$ fuera estadísticamente diferente a la que se obtuvo con la Solución Steiner. En la Tabla 4 también se puede observar que a los ocho días después del almacenamiento de frutos, los grados Brix fueron superiores (45,3\%) en aquellos producidos por plantas tratadas con solución Steiner, en comparación con el promedio estimado para los frutos de plantas sometidas al tratamiento $\mathrm{T}_{8}$.

Tabla 4. pH, acidez titulable y sólidos solubles totales de tomate saladette a los 0,8 y 16 días de almacenamiento a $20{ }^{\circ} \mathrm{C}$ y $85 \%$ de humedad relativa

\begin{tabular}{|c|c|c|c|c|c|c|c|c|c|}
\hline \multirow{3}{*}{ Trat. } & \multicolumn{3}{|c|}{$\mathrm{pH}$} & \multicolumn{3}{|c|}{ Acidez titulable } & \multicolumn{3}{|c|}{ Sólidos solubles totales $\left({ }^{\circ} \mathrm{B}\right)$} \\
\hline & \multicolumn{9}{|c|}{ Días } \\
\hline & 0 & 8 & 16 & 0 & 8 & 16 & 0 & 8 & 16 \\
\hline $\mathrm{T}_{1}$ & $4.49 \mathrm{a}^{*}$ & $4.48 \mathrm{ab}$ & $4.47 \mathrm{a}$ & $0.27 \mathrm{a}$ & $0.32 \mathrm{a}$ & $0.31 \mathrm{ab}$ & $4.02 \mathrm{a}$ & $4.62 \mathrm{a}$ & $5.22 \mathrm{a}$ \\
\hline $\mathrm{T}_{2}$ & $4.0 \mathrm{ab}$ & $4.51 \mathrm{a}$ & $4.56 \mathrm{a}$ & $0.28 \mathrm{a}$ & $0.30 \mathrm{a}$ & $0.30 \mathrm{ab}$ & $4.20 \mathrm{a}$ & $3.78 \mathrm{ab}$ & $4.80 \mathrm{a}$ \\
\hline $\mathrm{T}_{3}$ & $4.35 \mathrm{abc}$ & $4.45 a b$ & $4.58 \mathrm{a}$ & $0.28 \mathrm{a}$ & $0.35 \mathrm{a}$ & $0.32 \mathrm{a}$ & $3.78 \mathrm{a}$ & $4.02 \mathrm{ab}$ & $4.98 \mathrm{a}$ \\
\hline $\mathrm{T}_{4}$ & $4.29 \mathrm{bc}$ & $4.44 \mathrm{ab}$ & $4.48 \mathrm{a}$ & $0.28 \mathrm{a}$ & $0.36 \mathrm{a}$ & $0.32 \mathrm{a}$ & $3.60 \mathrm{a}$ & $4.20 \mathrm{ab}$ & $4.62 \mathrm{a}$ \\
\hline $\mathrm{T}_{5}$ & $4.28 \mathrm{bc}$ & $4.37 \mathrm{~b}$ & $4.52 \mathrm{a}$ & $0.31 \mathrm{a}$ & $0.36 \mathrm{a}$ & $0.29 \mathrm{ab}$ & $3.78 \mathrm{a}$ & $3.78 \mathrm{ab}$ & $4.38 \mathrm{a}$ \\
\hline $\mathrm{T}_{6}$ & $4.33 \mathrm{abc}$ & $4.47 \mathrm{ab}$ & $4.50 \mathrm{a}$ & $0.36 \mathrm{a}$ & $0.30 \mathrm{a}$ & $0.30 \mathrm{ab}$ & $3.78 \mathrm{a}$ & $3.78 \mathrm{ab}$ & $4.62 \mathrm{a}$ \\
\hline $\mathrm{T}_{7}$ & $4.33 \mathrm{abc}$ & $4.42 \mathrm{ab}$ & $4.52 \mathrm{a}$ & $0.32 \mathrm{a}$ & $0.33 \mathrm{a}$ & $0.25 \mathrm{~b}$ & $3.78 \mathrm{a}$ & $3.60 \mathrm{ab}$ & $3.78 \mathrm{a}$ \\
\hline $\mathrm{T}_{8}$ & $4.21 \mathrm{c}$ & $4.37 \mathrm{~b}$ & $4.51 \mathrm{a}$ & $0.37 \mathrm{a}$ & $0.34 \mathrm{a}$ & $0.25 \mathrm{~b}$ & $3.60 \mathrm{a}$ & $3.18 \mathrm{~b}$ & $4.20 \mathrm{a}$ \\
\hline $\mathrm{T}_{9}$ & $4.40 \mathrm{abc}$ & $4.44 \mathrm{ab}$ & $4.46 \mathrm{a}$ & $0.37 \mathrm{a}$ & $0.31 \mathrm{a}$ & $0.29 \mathrm{ab}$ & $4.02 \mathrm{a}$ & $4.02 \mathrm{ab}$ & $4.38 \mathrm{a}$ \\
\hline
\end{tabular}

*Medias con la misma letra en la columna no son significativamente diferentes. Tukey $(\mathrm{p} \leq 0,05)$.

De igual manera que respecto a la producción, en la calidad postcosecha de los frutos no fue perceptible la acción de los microorganismos adicionados (Tabla 2), ya que los promedios de $\mathrm{pH}$, acidez titulable y sólidos solubles fueron estadísticamente similares en todos los pares de tratamientos que incluyeron la misma cantidad de composta y MPA, y sólo discreparon en aquellos tratamientos con diferencias en la cantidad de microorganismos aplicados.

\section{6}

\section{DISCUSIÓN}

Con el tratamiento $\mathrm{T}_{8}$ se obtuvieron frutos extra grandes, grandes y medianos en una cantidad similar a la que se logró con la solución Steiner $\left(\mathrm{T}_{1}\right)$, lo que quizá pudo deberse a que con ambos tratamientos se proporcionaron similares nutrientes. Como lo menciona Lewin (1996), probablemente estos nutrientes fueron metabolizados en más compuestos orgánicos, como las enzimas necesarias para transcribir por más tiempo el material genético, entre otras, y formar más moléculas del ácido ribonucleico mensajero y, en consecuencia, generar más proteínas que forman parte de la materia seca que constituye los frutos. Lo anterior puede ser debido a que los procesos de mineralización de la composta y solubilización de los MPA ocurrieron a una velocidad tal que permitieron la aportación de nutrimentos en cantidades muy similares a lo aportado por la solución Steiner, ya que, como lo reportaron 
Eghball et al. (2002), al utilizar composta de estiércol de bovino, en el primer año la mineralización de nitrógeno fue de $18 \%$, mientras que cuando se utilizó composta de estiércol de cerdo o de gallina dicha mineralización fue del $55 \%$, en tanto que el potasio estuvo disponible en su totalidad, la mineralización del $\mathrm{Ca}$ y $\mathrm{Mg}$ fue de $55 \%$ y la de B, S, Cu, Mn y Zn fue de $40 \%$. En este sentido, el potencial de la combinación de materiales orgánicos junto con los fertilizantes de roca en las estrategias de reposición de la fertilidad del suelo se acentúa (Van, 2006).

La mayor producción de frutos chicos se obtuvo en las parcelas cultivadas con aquellos tratamientos diferentes a $\mathrm{T}_{8}$ y la solución Steiner, lo que a su vez se puede atribuir a la insuficiente disponibilidad de nutrimentos metabolizados para formar la materia seca, alcanzando solamente para inducir ese tamaño de frutos, ya que de otra manera las plantas hubieran formado frutos extra grandes, grandes y medianos, en promedios similares a los que se lograron con $\mathrm{T}_{8}$ y la solución Steiner, puesto que aparte de los tratamientos, las plantas fueron del mismo genotipo y todas las parcelas fueron manejadas de la misma manera. Esto indica que las cantidades de los fertilizantes utilizados, diferentes a $\mathrm{T}_{8}$ y la solución Steiner, no fueron las adecuadas para inducir más metabolismo de nutrimentos en sustancias orgánicas y, por tanto, más producción de frutos extra grandes, grandes y medianos, como lo mencionan Rotenberg et al. (2007), al plantear que los fertilizantes orgánicos ejercen un efecto multilateral sobre las propiedades agronómicas de los suelos y, cuando se utilizan correctamente, elevan de manera adecuada la cosecha de los cultivos. A esto último hay que añadir que a partir de la vermicomposta se generan ácido indolacético, giberelinas y citoquininas, los cuales ocasionan efectos significativos en el crecimiento de las plantas, toda vez que pueden ser adsorbidos en los humatos y actuar en conjunto con ellos sobre dicho crecimiento (Atiyeh et al., 2002). Por todo lo anterior, como lo dicen Ruiz et al. (2009), con los bioproductos que se generan a partir de fertilizantes orgánicos es posible inducir buen crecimiento y desarrollo de plantas con bajas aplicaciones de fertilizantes de origen industrial. Además, como lo indica Hallmann (2012), los frutos de tomate que se producen bajo sistema orgánico contienen alto nivel de antioxidantes, vitamina $\mathrm{C}$, polifenoles (incluso flavonoides) y carotenoides (como el lycopeno y ß-caroteno).

La producción total $\left(113,97 \mathrm{Mg} \mathrm{a}^{-1}\right)$ que se obtuvo de las parcelas donde se aplicó $\mathrm{T}_{8}\left(25 \mathrm{Mg} \mathrm{ha}^{-1}\right.$ de composta
+ $6 \mathrm{Mg} \mathrm{ha}^{-1}$ de MPA) no sólo superó a la que se cosechó en aquellas parcelas en las que aplicaron otras dosis de fertilizantes que incluyeron composta, MPA y M, sino que también fue superior a los rendimientos reportados por Preciado et al. (2011), que fueron de 12,8, 6,09, 10,16 y $5,46 \mathrm{~kg} \mathrm{~m}^{2}$, cuando cultivaron tomate saladette con solución Steiner, té de composta, té de vermicomposta y lixiviado de vermicomposta, respectivamente. También superó la producción que obtuvieron Cun et al. (2008), al combinar el biofertilizante EcoMic ${ }^{\circledR}$ (hongos micorrizógenos del género Glomus) y el humus de lombriz, obteniendo $8,4 \mathrm{~kg} \mathrm{~m}^{2}$. Asimismo, se superó el rendimiento informado por De la Cruz et al. (2009), quienes obtuvieron $39,8 \mathrm{Mg} \mathrm{ha}^{-1}$, al aplicar composta generada de estiércol de bovino, rastrojo de maíz, zacate elefante y tierra negra (CEMZT) al $75 \%+$ arena y la vermicomposta de estiércol, pasto bahía y tierra negra (VEPT) al 100 y $50 \%$ + arena. La producción que se obtuvo en esta investigación también fue superior a la que reportaron De la Cruz et al. (2010), quienes con la mezcla (1:1:1, V:V:V) de vermicomposta de estiércol de ganado vacuno + rastrojo de maíz y tierra negra (75 \% + $25 \%$ de arena) obtuvieron 57,4 $\mathrm{Mg} \mathrm{ha}^{-1}$ de tomate.

La producción resultante de este experimento fue inferior a la que reportaron Ortega et al. (2010), al cultivar tomate ( 6 plantas $\mathrm{m}^{2}$ ) en sustrato formado por aserrín de pino composteado + composta de estiércol de ovino y solución Steiner, con lo que se obtuvo una producción total de $25 \mathrm{~kg} \mathrm{~m}^{-2}$. También fue inferior a la producción reportada por Cruz et al. (2012), quienes obtuvieron 146,33 $\mathrm{Mg} \mathrm{ha}^{-1}$ al cultivar el híbrido 'Charleston' en una mezcla de tezontle + vermicomposta de estiércol bovino y desechos vegetales. Márquez et al. (2013) al usar las variedades de tomate Big Beef y Bosky cultivadas con una solución inorgánica y composta + macro y micro elementos inorgánicos, reportaron una producción promedio de 136,7 Mg ha $^{-1}$, superando también la producción obtenida en la presente investigación. La influencia del consorcio microbiano no fue perceptible, quizás porque la intensidad del metabolismo de nutrientes en las plantas de tomate no requirió de mayor cantidad de nutrimentos que aquella que se logró con la mineralización en el bocashi y MPA.

Con respecto a la calidad postcosecha, para que los frutos de tomate tengan buen sabor y se facilite su industrialización, el pH debe ser de 4,4 (Hidalgo et al., 1998), de tal forma que los resultados de esta investigación indican que los frutos obtenidos después de la aplicación de solución Steiner, composta, MPA 
y $\mathrm{M}$, cumplen con este requerimiento. Además, estos resultados coinciden con los promedios reportados por Aldrich et al. (2010), quienes al medir pH en frutos de tomate de diferentes cultivos en sistemas de producción orgánica reportaron valores entre 4,2 y 4,4, coincidentes con los reportados por Vinha et al. (2014), quienes al medir el pH de frutos que se manejaron bajo sistema de producción orgánica y convencional obtuvieron lecturas de 4,38 y 4,46, respectivamente. Asimismo, Migliori et al. (2012) encontraron valores de 4,27 y 4,30. Esto último deja entrever que la expresión del $\mathrm{pH}$ de los frutos es una característica que se debe a la interacción genotipo-ambiente.

La acidez titulable de los frutos de tomate a los 16 días de almacenamiento coincide con lo reportado por Navarro et al. (2012), quienes usando agua residual y de pozo para la producción de tomate y almacenando los frutos a diferentes temperaturas y porcentajes de humedad relativa durante $0,10,20,30$ y 40 días, encontraron que a los 20 días dicha variable presentó diferencias estadísticamente significativas, reportando valores similares a los registrados en esta investigación. Peña et al. (2013) evaluaron sustratos a base de cascarilla cruda, cascarilla quemada en mezcla con escoria de carbón, fibra de coco y zeolita, cascarilla cielo abierto con escoria y fibra de coco con zeolita. Los registros de acidez titulable reportados fluctuaron entre 0,3 y 0,38 $\%$, lo que concuerda con los datos reportados en esta investigación.

En cuanto a sólidos solubles totales, De la Cruz et al. (2010) mencionan que al aplicar vermicomposta, entre otros sustratos, se incrementa el contenido de dichos sólidos en tomate, y que este carácter oscila entre 4,63 y 4,96 ${ }^{\circ}$ Brix. Por su parte Aldrich et al. (2010) reportan datos que fluctúan entre 3,52 y $4,79^{\circ}$ Brix, lo que a su vez concuerda con los resultados de esta investigación. Resultados semejantes reportaron Preciado et al. (2011), quienes evaluaron la producción de tomate cultivado con solución Steiner, té de composta, té de vermicomposta y lixiviado de vermicomposta, encontrando valores de 4,$1 ; 4,4 ; 4,5$ y $4,6^{\circ}$ Brix, respectivamente. Ochoa et al. (2009) evaluaron cuatro fuentes de nitrógeno aplicados en arena: a) fertilización inorgánica con solución nutritiva en sustrato de arena, b) té de composta en sustrato de arena, c) té de composta diluido, en una relación té: agua de 1:3, aplicado en un sustrato de arena + composta en proporción 1:1 en volumen y d) incorporación gradual de la mezcla arena + composta:
$50 \%$ del volumen de la maceta al inicio, $25 \%$ a los 75 días después del trasplante (ddt) y el $25 \%$ restante a los 150 ddt. Para estas cuatro fuentes de nitrógeno, estos autores encontraron valores de 3,71, 4,41, 4,33 y $4,59{ }^{\circ}$ Brix, respectivamente. Por su parte Rodríguez et al. (2009) estudiaron el sustrato arena: composta (1:1; v:v) + té de composta diluido (1:3; v:v) y registraron $4,5^{\circ}$ Brix en los frutos de tomate. Otros factores que influyen en la producción de tomate son la interacción compleja de efectos ambientales, el método de cultivo y la selección de cultivares que influyen en la calidad y las propiedades antioxidantes de los tomates, lo que es fundamental para la producción de alimentos frescos de alta calidad (Aldrich et al., 2010).

\section{CONCLUSIONES}

Con el híbrido 'Moctezuma' y la fertilización de ésta con

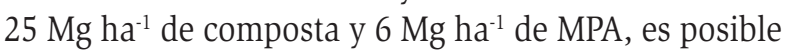
obtener una producción similar a la que se puede lograr con solución nutritiva Steiner, sin que en los frutos se alteren las propiedades de $\mathrm{pH}$, acidez titulable y sólidos solubles.

\section{AGRADECIMIENTOS}

Al Consejo Nacional de Ciencia y Tecnología y Universidad Autónoma de Sinaloa. Al Centro de Investigación en Alimentación y Desarrollo Unidad Culiacán, en particular a la Dra. María Dolores Muy Rangel y MC Laura Aracely Contreras Angulo, por su apoyo al presente trabajo. Al Colegio de Michoacán, extensión La Piedad, por los análisis realizados.

\section{BIBLIOGRAFÍA}

Aldrich, H.T., K. Salandanan, P. Kendall, M. Bunning, F. Stonaker, O. Külen y C. Stushnoff. 2010. Cultivar choice provides options for local production of organic and conventionally produced tomatoes with higher quality and antioxidant content. Journal of the Science of Food and Agriculture 90: 2548-2555.

AOAC. 1975. Oficial Methods of Analysis of the Association of official Analytical Chemists. 12th edition. Washington, USA. 354 p.

Atiyeh, B. S., R. Dham, M. Kadry, A. F. Abdallah, M. AlOteify, O. Fathi y A. Samir. 2002. Benefit cost analysis of moist exposed burn ointment. Burns 28: 659-663. 
Cruz, C. E., V. M. Sandoval, H. V. H. Volke, C. A. Can y E. J. Sánchez. 2012. Efecto de mezclas de sustratos y concentración de la solución nutritiva en el crecimiento y rendimiento de tomate. Revista Mexicana de Ciencias Agrícolas 3(7): 1361-1373.

Cun, G. R., D. C. Duarte y S. L. Montero. 2008. Producción orgánica de tomate mediante la aplicación de humus de lombriz y $\mathrm{EcoMic}^{\circledR}$ en condiciones de casa de cultivo. Revista Ciencias Técnicas Agropecuarias 17(3): 22-25

Da Silva, R. L., A. R. Dos Santos, S. L. F. Silva y J. S. Souza. 2010. Rochas silicáticas portadoras de potássio como fontes do nutriente para as plantas solo. Revista Brasileira de Ciência do Solo 34: 891-897.

De la Cruz, L. E., E. M. A Botello, T. V. Robledo, O. R. Osorio, H. C. Márquez y H. R. Sánchez. 2009. Producción de tomate en invernadero con composta y vermicomposta como sustrato. Universidad y Ciencia 25(1): 59-67.

De la Cruz, L. E., O. R. Osorio, M. E. Martínez, A. J. Lozano del Rio, V. A. Gómez y H. R. Sánchez. 2010. Uso de compostas y vermicompostas para la producción de tomate orgánico en invernadero. Interciencia 35(5): 363-368.

Eghball, B., B. J. Wienhold, J. E. Gilley y R. A. Eigenberg. 2002. Mineralization of Manure Nutrients. Journal of Soil and Water Conservation 57(6): 469-473.

García, A. E. 1988. Modificaciones al sistema de clasificación climática de Köppen. Instituto de Geografía. UNAM, México 217 p.

García, Y., W. Ramírez y S. Sánchez. 2012. Indicadores de la calidad de los suelos: Una nueva manera de evaluar éste recurso. Revista Pastos y Forrajes. Matanzas, Cuba. 35(2): 125-138.

Guelfi, S. D. R., G. Marchi, C. R. Spehar, G. L. R. Guimarães, T. A. Rein, S. D. Araujo y F. W. Ávila. 2012. Characterization and nutrient release from silicate rocks and influence on chemical changes in soil. Revista Brasileira de Ciência do Solo 36: 951-962.

Hallmann, E. 2012. The influence of organic and conventional cultivation systems on the nutritional value and content of bioactive compounds in selected tomato types. Journal of the science of food and agriculture 92(14): 2840-2848.

Hidalgo, G. J. C., G. G. Alcántar, C. G. A. Baca, G. P. Sánchez y E. J. A. Escalante. 1998. Efecto de la condición nutrimental de las plantas y de la composición y calidad en tomate. Terra Latinoamericana 16(2): 143-148.
Lewin, B. 1996. Genes. Ed. Reverté. S.A. Barcelona, España, 613 p.

Márquez, H. C., R. P. Cano, V. U. Figueroa, D. J. A Avila, D. N. Rodríguez y H. J. L. García. 2013. Yield and quality of tomato with organic sources of fertilization under greenhouse conditions. International Jurnal of Experimental Botany 82: 55-61.

Migliori, C., L. F. D. Cesare, R. L. Scalzo, G. Campanelli y V. Ferrari. 2012. Effects of organic farming and genotype farming and genotype on alimentary and nutraceutical parameters in tomato fruits. Journal of the Science of Food Agriculture 92(14): 2833-2839.

Navarro, L. E. R., A. R. Nieto, G. J. Corrales, M. M. R. García y A. A. Ramírez 2012. Calidad poscosecha en frutos de tomate hidropónico producidos con agua residual y de pozo. Revista Chapingo. Serie Horticultura 18(3): 263-277.

Nicholls, C. I y M. A. Altieri. 2012. Modelos ecológicos y resilientes de producción agrícola para el siglo XXI. Agroecología 6: 28-37.

Nieto, G. A., A. B. Murillo, D. E. Troyo, M. J. A Larrinaga, y H. J. L García. 2002. El uso de compostas como alternativa ecológica para la producción sostenible del chile (Capsicum annuum L.) en zonas áridas. Interciencia 27(8): 417-421.

Ochoa C. D. C y R. A. Montoya. 2010. Consorcios microbianos: una metáfora biológica aplicada a la asociatividad empresarial en cadenas productivas agropecuarias. Revista Facultad de Ciencias Económicas: Investigación y Reflexión 18(2): 55-74.

Ochoa, M. E., V. U. Figueroa, R. P. Cano, R. P. Preciado, R. A. Moreno y D. N. Rodríguez. 2009. Té de composta como fertilizante orgánico en la producción de tomate (Lycopersicon esculentum Mill.) en invernadero. Revista Chapingo. Serie Horticultura 15(3): 245-250.

Olivares, C. M. A., R. A. Hernández, C. C. Vences, B. J. L. Jáquez y B. D.Ojeda. 2012. Worm compost and dairy cattle manure compost as fertilisers and in soil improvement. Universidad y Ciencia 28(1): 27-37.

Ortíz, F. P y A. J. P Amado, 2003. Uso de biofertilizantes en avena de temporal en la sierra de Chihuahua. Sociedad Mexicana de la Ciencia del Suelo A.C. 174-191.

Ortega, M. L. D., O. J. Sánchez, M. J. Ocampo, C. E. Sandoval, R. B. A. Salcido y R. F. Manzo. 2010. Efecto de diferentes sustratos en crecimiento y rendimiento de tomate (Lycopersicum esculentum Mill.) bajo condiciones de invernadero. Ra Ximhai 6(3): 339-346. 
Peña, M. Y., F. Casierra-Posada, y O. I. Monsalve. 2013. Producción hidropónica de tomate (Solanum lycopersicum L.) en cascarilla de arroz mezclada con materiales minerales y orgánicos. Revista Colombiana de Ciencias Hortícolas 7(2): 217-227

Preciado, R. P., M. Fortis, J. L. García-Hernández, E. O. Rueda, J. R. Esparza, A. Lara, M. A. Segura y J. A. Orozco. 2011. Evaluación de soluciones nutritivas orgánicas en la producción de tomate en invernadero. Interciencia 36(9): 689-693.

Rodríguez, D. N., P. Cano, U. Figueroa, E. Favela, A. Moreno, C. Márquez, E. Ochoa y P. Preciado. 2009. Uso de abonos orgánicos en la producción de tomate en invernadero. Terra Latinoamericana 27: 319-327.

Rotenberg, D., A. Jiménez, E. J. Chapman, A. E. Whitfield, R. M. Goodman y L. R. Cooperband. 2007. Soil properties associated with organic matter-mediated suppression of bean root rot in field soil amended with fresh and composted paper mill residuals. Soil Biology \& Biochemistry 39: 2936-2948.

Ruiz, J., E. Terry, T. Tejeda y M. M. Díaz. 2009. Aplicación de bioproductos a la producción ecológica de tomate. Cultivos Tropicales 30(3): 60-64.

SAS. Institute .1985. SAS User's Guide, Básics, Versión 5 Edition. SAS Institute Inc., Cary, N.C 1181-1191 p.
Stamford, N. P., P. M. Moura, J. M. A Lira, C. E. de R. S. Santos, L. H. Duenhas y C. A. Gava. 2009. Chemical attributes of an Argisoil of the Vale do São Francisco after melon growth with phosphate andpotash rocks biofertilizers. Horticultura Brasileira 27: 447-452.

Steiner, A. A. 1961. A Universal method for preparing nutrient solutions of a certain desired composition Plant Soil. 134-154.

USDA. 1992. Standards for grades of fresh tomatoes. Washington DC, USA. 76 pp.

Van, S. P. 2006. Farming with rocks and minerals: challenges and opportunities. Anais da Academia Brasileira de Ciências 78(4): 731-747.

Villarreal, R. M., S. Hernández, P. Sánchez, R. S. García, T. Osuna, S. Parra, y A. D Armenta. 2006. Effect of Legume Cover Crops on TomatoYield and Quality. Terra Latinoamericana 24 (4):549-556.

Vinha, A. F., S. V. Barreira, A. S. Costa, R. C. Alves y M. B. Oliveira. 2014. Organic versus conventional tomatoes: Influence physicoqhemical parameters, bioactive compounds and sensorial attributes. Food and chemical toxicology. 67:139-144.

Para citar este artículo: Parra-Delgado, J. M., T. J. Velázquez-Alcaraz, E. Quero-Gutiérrez, L. Partida-Ruvalcaba, T. Díaz-Valdés, B. Galván-Piña y F. Ayala-Tafoya. 2014. Uso de composta, minerales primarios amorfos y microrganismos para la producción y calidad de tomate. Revista Intropica 9: 102 - 110 